Revue d'histoire du XIXe siècle

Société d'histoire de la révolution de 1848 et des

révolutions du XIXe siècle

56 | 2018

Un autre $\mathrm{XIX}^{\mathrm{e}}$ siècle : I'Inde sous domination coloniale

\title{
Technologie et transfert de technologie dans l'Empire britannique en Inde
}

Ian J. Kerr

Traducteur : Laurent Bury

\section{OpenEdition}

\section{Journals}

Édition électronique

URL : http://journals.openedition.org/rh19/5533

DOI : $10.4000 /$ rh19.5533

ISSN : $1777-5329$

Éditeur

La Société de 1848

Édition imprimée

Date de publication : 15 octobre 2018

Pagination : 134-137

ISSN : 1265-1354

Référence électronique

Ian J. Kerr, "Technologie et transfert de technologie dans l'Empire britannique en Inde », Revue d'histoire du XIXe siècle [En ligne], 56 | 2018, mis en ligne le 15 octobre 2020, consulté le 04 janvier 2021. URL : http://journals.openedition.org/rh19/5533; DOI : https://doi.org/10.4000/rh19.5533 
dirigeant a visité de long en large l'Europe, l'Amérique du Nord, l'Afrique et l'Asie de l'Est. Il a profité de ces tournées pour rencontrer et apporter un soutien financier à des éléments politiquement actifs de la diaspora indienne, du leader du Congrès national indien basé à Londres, Dadabhai Naoroji, aux figures révolutionnaires en exil telles que Bhikaji Cama et Shyamji Krishnavarma. Loin d'être une île de tradition, Baroda était connectée aux réseaux de personnes, d'idées et de ressources qui soudaient l'Empire britannique tout en contribuant à sa disparition potentielle. Bien que Baroda ne soit pas représentatif des États princiers dans leur ensemble, certaines de ses caractéristiques politiques sont présentes ailleurs. Les dirigeants d'autres États de l'Inde occidentale - en particulier Kolhapur - se sont également engagés dans des politiques anticoloniales au-delà de leurs frontières.

De récentes critiques ont mis en évidence les dangers du «tournant global» en histoire, qui consiste à présenter des connexions anciennes comme quantitativement et qualitativement continues, ignorant les hauts et les bas et ne formulant pas de cadres explicatifs complets ${ }^{22}$. Alors que le tournant global a peut-être atteint ce que David A. Bell a qualifié de "point de rendements décroissants" dans d'autres domaines, il conserve sa valeur pour l'étude des États princiers indiens ${ }^{23}$. L'approche des États à travers le tournant global nous permet de les considérer comme une partie constitutive de l'Inde et de l'Empire britannique, soulignant leur position ambivalente en tant que contreforts du pouvoir impérial et agents de décolonisation. Cette enquête, qui réétalonne notre compréhension des limites de l'Empire britannique, peut faire de même pour d'autres projets impériaux contemporains, où les formes locales de royauté et d'autorité coexistaient avec les régimes coloniaux.

\section{Teresa Segura-Garcia est chercheuse Juan de la Cierva à l'université Pompeu Fabra, à Barcelone}

\section{IAN J. KERR \\ Technologie et transfert de technologie dans l'Empire britannique en Inde}

$\mathrm{Au} \mathrm{XIX}^{\mathrm{e}}$ siècle, les partisans de la présence impériale britannique sur le sous-continent indien et ailleurs pensaient que la technologie permettrait de renforcer l'autorité coloniale et de favoriser les intérêts de la Grande-Bre-

22. Samuel Moyn et Andrew Sartori, "Approaches to global intellectual history”, in Samuel Moyn et Andrew Sartori (eds), Global intellectual history, New York, Columbia University Press, 2015, p. 3-32 (p. 21-23).

23. David A. Bell, "This is what happens when historians overuse the idea of the network", New Republic, <https://newrepublic.com/article/114709/world-connecting-reviewed-historiansoveruse-network-metaphor> [consulté le $1^{\text {er }}$ décembre 2017] 
tagne. Ils croyaient aussi fermement que le transfert de technologies comme le chemin de fer vers les colonies déclenchait des changements nécessairement positifs pour les peuples soumis par l'empire britannique. Comme un orateur le dit en 1853, lorsqu'une ligne de chemin de fer fut ouverte dans l'ouest de l'Inde: «Le chemin de fer, installé par des hommes capables et prudents, serait le plus puissant de tous les instruments terrestres pour le progrès de la civilisation à tous points de vue $[\ldots] »^{24}$.

Progrès ou pas? Les avis sur ce point sont divergents et relèvent en grande partie du jugement de valeur. Il est cependant indiscutable que tout un ensemble de technologies - allant de systèmes techniques complexes et de grande taille comme le chemin de fer à des objets spécifiques comme la mitrailleuse Gatling ou à l'usage prophylactique de la quinine contre le paludisme - facilita l'expansion, l'entretien et l'exploitation des possessions impériales britanniques en Inde et ailleurs.

Ces conséquences ont poussé certains historiens, notamment Daniel R. Headrick, à attribuer à la technologie un rôle central en ce qu'elle a rendu possible l'expansion impériale, et rendu caducs les outils, machines et techniques indigènes à mesure que s'implantaient les technologies "supérieures " transférées depuis l'Occident ${ }^{25}$. Dans un ouvrage plus récent (2012), Headrick s'est orienté vers une formulation plus "soft» du rôle de la technologie dans l'histoire, en reconnaissant que «les technologies sont toujours spécifiques à un environnement. Bien qu'elles offrent à leurs détenteurs un pouvoir sur la nature, ce pouvoir est limité à certaines parties spécifiques de la nature $»^{26}$.

Ce même livre inclut néanmoins un chapitre sur l'impérialisme des bateaux à vapeur (navires à faible tirant d'eau, utilisés surtout sur les fleuves et le long des côtes), qui insiste sur l'efficacité de cette technologie pour établir l'impérialisme occidental dans toutes sortes de régions.

Cet argument, et en particulier son application à l'expansion britannique en Birmanie et en Afrique occidentale, a été sévèrement critiqué par Clive Dewey, connu de longue date pour son éminente contribution à l'histoire

24. Cité in Ian J. Kerr, Engines of Change. The Railroads That Made India, Westport, Praeger, 2007, p. 6

25. The Tools of Empire. Technology and European Imperialism in the Nineteenth Century, New York, Oxford University Press, 1981, et The Tentacles of Progress. Technology Transfer in the Age of Imperialism, 1850-1940, New York, Oxford University Press, 1988.

26. Daniel R. Headrick, Power Over Peoples. Technology, Environments, and Western Imperialism, 1400 to the Present, Princeton, Princeton University Press, 2010, p. 370. Les chercheurs qui étudient le rôle transformateur de la technologie se situent à l'intérieur d'un continuum allant du déterminisme dur, où la technologie est une force autonome induisant des changements, jusqu'à différentes versions $\mathrm{du}$ déterminisme soft, où la technologie est une variante significative mais dépendante, parmi tant d'autres dans le flux contextualisé de l'existence humaine. Cette extrémité du spectre est la plus peuplée. Un examen attentif révèle très peu d'adeptes d'un déterminisme dur sans mélange. C'est la pensée "populaire" plutôt que le discours savant qui accueille ce déterminisme dur. Pour une exploration vigoureuse et diversifiée de ces questions, lire M. R. Smith et L. Marx [dir.], Does Technology Drive History? The Dilemma of Technological Determinism, Cambridge, Mass., The MIT Press, 1994. 
de l'Asie coloniale ${ }^{27}$. Le livre de Dewey propose un exposé magistral du coûteux échec essuyé par les autorités coloniales sur plusieurs décennies (de 1839 à 1878 environ) dans leur tentative de création d'une flotte de bateaux à vapeur sur les eaux mouvantes et fluctuantes de l'Indus et de ses principaux affluents. À l'opposé de cet échec se situe la réussite durable de la technologie «traditionnelle» précoloniale, des divers bateaux propulsés par l'énergie humaine et commandés par des marins ayant une profonde connaissance des caprices des rivières sur lesquels voguaient leurs embarcations simples mais efficaces.

Il s'agissait au fond d'un échec technique : il était alors impossible de construire des navires à vapeur satisfaisant les multiples critères nécessaires pour une navigation sur l'Indus qui soit efficace, durable et économiquement viable. Pourtant, ce qui frappe dans cette histoire, c'est l'opiniâtreté dispendieuse des autorités qui persistèrent dans leur désir de monter une flottille à vapeur malgré ces échecs répétés et face au succès des bateaux indigènes. Selon leur idéologie, les machines occidentales devaient forcément permettre de maîtriser l'Indus ${ }^{28}$. Dans la terminologie de Headrick, un contexte spécifique à un environnement créait des obstacles que la technologie du $\mathrm{XIX}^{\mathrm{e}}$ siècle ne pouvait surmonter, comme il le reconnaît indirectement dans sa brève mention des navires à vapeur de l'Indus ${ }^{29}$.

S'appuyant sur une reconstitution minutieuse et convaincante, Dewey lance une vaste et virulente attaque contre ce qu'il appelle le «millénarisme technologique» en histoire impériale, une approche fondée sur les «outils de l'empire» et qui, selon lui, repose sur trois postulats erronés : (1) les technologies occidentales transférées étaient librement choisies par les autorités occidentales sans consulter aucun autre avis; (2) lesdites technologies consolidaient systématiquement le pouvoir colonial; (3) elles détruisaient les techniques traditionnelles (c'est-à-dire préexistantes). Ces postulats me semblent erronés, et ils ont été clairement réfutés par bon nombre de travaux récents.

En dénonçant de façon claire, bien qu'un peu polémique, les cadres interprétatifs déficients, Dewey nous rend service à tous. L'approche «outils de l'empire» n'est sans doute pas au-dessus de tout soupçon. Et pourtant, toutes sortes de technologies ont parfois facilité l'expansion, l'entretien et l'exploitation de l'empire britannique. Tout cela - comme Headrick le reconnaissait en 2010 - s'est produit de manière variable, dans des environnements et des contextes spécifiques. Pour être équitable envers Headrick et d'autres, il faut aussi admettre que des tensions et parfois des erreurs peuvent apparaître dans

27. Steamboats on the Indus. The Limits of Western Technological Superiority in South Asia, New Delhi, Oxford University Press, 2014, en particulier p. 246-248.

28. Je fais ici référence au complexe d'attitudes et d'actions savamment exploré par Michael Adas, Machines as the Measure of Men. Science, Technology, and Ideologies of Western Dominance, Ithaca, Cornell University Press, 1989.

29. Headrick, Power Over Peoples, op. cit., p. 188. 
les ouvrages de synthèse, face aux détails et aux reconstitutions minutieuses que permet une étude de cas.

En ce qui concerne les chemins de fer, très peu d'historiens affirmeraient en 2018 qu'ils en vinrent très vite, à la fin du XIX ${ }^{\mathrm{e}}$ siècle, à dominer de façon décisive le transport terrestre dans toute l'Inde ${ }^{30}$. Les changements prirent du temps, des décennies, voire davantage, et le triomphe du chemin de fer, toujours lié au contexte, fut inégal et souvent incomplet - autant de points bien illustrés par Dewey dans sa fine description de la concurrence entre marins indigènes et chemins de fer au Pendjab et dans le Sind. De plus, une certaine hybridité était présente dans la construction des chemins de fer indiens et les Asiatiques jouèrent un rôle important dans les processus de transfert de technologie. Plus généralement, les approches actuelles de la relation entre impérialisme et technologie mettent en avant les notions d'hybridité, d'activité, d'adaptation, les spécificités contextuelles, et les interactions entre colonies et métropoles, entre colonies et colonies, entre colons et colonisés ${ }^{31}$.

La locomotion à vapeur - navires à vapeur sur les mers et certains fleuves, locomotives à vapeur sur les rails - associée à de meilleurs ports et au Canal de Suez à partir de 1869, n'a pas crée l'Empire britannique en Inde, mais elle a facilité l'entretien et l'exploitation de cet empire (pas sur l'Indus, toutefois). Sur le long terme, elle a eu des conséquences imprévues qui ont sapé l'autorité britannique. Sur ce dernier point, je suis entièrement d'accord avec Dewey.

Ian J. Kerr est Senior scholar à l'université du Manitoba, au Canada Traduit de l'anglais (Canada) par Laurent Bury

\section{LISE GUILHAMON \\ Les confluents de l'histoire et de la fiction dans la trilogie de l'Ibis d'Amitav Ghosh}

Si les thèmes de la mondialisation et de la migration sont, depuis les années 1980, une source d'inspiration majeure pour nombre d'écrivains d'origine indienne écrivant en anglais, aucun auteur n'a cependant eu autant à cœur d'explorer les racines historiques de ces phénomènes qu'Amitav Ghosh. Né à Calcutta en 1956, il obtient sa licence d'histoire à St Stephen's College à Delhi, puis étudie l'anthropologie sociale à Oxford. L'originalité de sa production littéraire tient au fait que c'est dans ces disciplines académiques mêmes que Ghosh puise, encore aujourd'hui, l'essentiel de son inspiration

30. Ian J. Kerr, Engines of Change, op. cit; Nitin Sinha, Communication and Colonialism in Eastern India. Bihar, 1760s-1880s, Londres, Anthem Press, 2012.

31. Pour une bonne vue d'ensemble, lire David Arnold, «Europe, technology, and colonialism in the 20th century", History and Technology, 21:1 (2005), p. 85-106. 